\title{
PRESENTACIÓN
}

\section{COMUNICACIÓN PARA IMAGINAR FUTUROS DESEABLES EN AMÉRICA LATINA}

\section{PROVOCACIONES DESDE EL SUR GLOBAL}

Una imagen que viajó en cuestión de segundos desde Bogotá (Colombia) a la serranía cordobesa (Argentina) hace pocas semanas resume en buena medida el espíritu actual de esta presentación. Es un diálogo entre los reconocidos Doc y Marty McFly, protagonistas de la película Volver al futuro. La leyenda que acompañaba dicha publicación sostenía: "Marty, pase lo que pase, NO VAYAS al 2020". Si bien el filme data de 1985, su actualidad es manifiesta. Entonces aparece la pregunta: ¿es este, a propósito del título del dosier, el futuro que imaginábamos? Creemos que no, ni por asomo comenzamos el año 2020 con la expectativa de una pandemia global ni del mundo tal como lo conocíamos. Un ejercicio en retrospectiva parece acertado: ¿no leímos las señales? ¿Las hubo? ¿Subestimamos la situación? ¿Qué hicimos con las noticias que venían desde China? ¿Pensamos que a nosotros, hijos del Abya Yala, no nos iba a pasar? ¿Veíamos con extrañeza a otras poblaciones, que sentíamos lejanas, construir hospitales en cuestión de semanas, trasladarse con tapabocas por las calles, vimos cómo de a poco hicieron el movimiento contrario, es decir, comenzaron a confinarse en sus hogares? Es una buena forma de describirlo: el futuro puede ser entendido en ese movimiento de repliegue y avance.

De a poco, nuestras cotidianidades y las de nuestros seres queridos empezaron a modificarse. Con aislamiento social, sin contacto físico, replegados de nuestros lugares habituales de trabajo, el paisaje vaciado de las universidades y centros de estudios. Las desigualdades se volvieron más explícitas en el continente más desigual de todos. Desigualdades porque no todos tienen una vivienda para hacer la cuarentena, ni acceso al agua potable para lavarse las manos y el rostro varias veces al día, o una computadora para seguir con las clases en modalidad virtual. Este futuro que ya llegó sabe a tendencias estructurales que, más allá de insostenidos esfuerzos gubernamentales y del rol cada vez más activo de la sociedad civil, cuesta revertir.

En este nuevo contexto nos preguntamos: ¿cuál es el escenario apuesta para la comunicación, entendida en su sentido más llano de "comunidad" y "puesta en común"? ¿Cómo será la comunicación del futuro que ya llegó, signado por nuevos modos de ser y estar juntos? 
Estos interrogantes no estaban en la imaginación de las editoras al proponer el tema del dosier, así como tampoco en el horizonte de los autores que escribieron ni del equipo editorial de la revista. Sin embargo, "Comunicación para imaginar futuros deseables en América Latina" puede ser parte de un escenario que apuesta desde la investigación, la docencia, los estudios de posgrado, el trabajo con las comunidades; es decir, desde nuestras prácticas cotidianas anteriores, aunque de manera reinventada.

Si decimos "imaginación”, podemos recurrir a la clásica y vigente definición del sociólogo norteamericano Charles W. Mills (1959), para quien la imaginación sociológica permite "comprender el escenario histórico más amplio en cuanto a su significado para la vida interior y para la trayectoria exterior de la diversidad de individuos", es "captar la historia y la biografía y la relación entre ambas dentro de la sociedad". Es, en definitiva, la "capacidad de pasar de una perspectiva a otra", de las "transformaciones más impersonales a las características más íntimas del yo humano" (pp. 26-27). La imaginación, por tanto, vincula la biografía y la historia; otorga capacidad de comprensión sobre los acontecimientos al establecer relaciones amplias, asignando sentido al lugar que vamos teniendo en la época que vivimos. Para nosotras, como investigadoras del campo de la comunicación, implicó la tarea de tentar a otros interesados en la labor de imaginar -es decir, vincular sus propias biografías con los hechos de los que forman parte- futuros deseables, escenarios apuesta para y con las poblaciones de América Latina. ¿Cómo nos fue en esa invitación? Recordamos brevemente un pasaje de la convocatoria al dosier:

Para Antonio Pasquali, el devenir pasa por comunicar en sociedades diversas con inclusión, equidad y democracia, porque "solo la diversidad es fecunda".

En tanto el argentino Gustavo Cimadevilla, actual presidente de ALAIC, señalaba: “La comunicación puede estar en el medio, antes o después, dentro o afuera, pero nunca ajena a las tensiones que esas luchas implican y que las tensiones humanas sostienen".

Si asumimos que el futuro es un ejercicio de construcción colectiva y que involucra a diversos actores, la comunicación puede pensarse como el escenario o territorio que posibilita el encuentro para consensuar o debatir tales visiones. En ese sentido, nos preguntamos: ¿qué futuros se están pensando/imaginando en nuestro continente? ¿Qué actores lideran esas visiones? ¿Está la comunicación acompañando, mediante acciones profesionales o de generación de conocimiento, esos procesos de construcción colectiva? ¿Hay visiones de futuro en tensión? ¿Hay futuros impuestos? ¿Consensuados? ¿Visibilizados? ¿Invisibilizados? (Kenbel y García Corredor, 2019)

A partir de estos interrogantes recibimos contribuciones provenientes de Argentina, Uruguay, Brasil, Colombia y Alemania. Contribuciones de investigadores, estudiantes de posgrado, docentes y periodistas con preocupaciones diversas. El modo en que organizamos su presentación léase también como el proceso mediante el cual los autores y las editoras pensamos, reflexionamos e imaginamos los futuros deseables. 
Las coordenadas para seguir el dosier llevan al encuentro del lector tres ejes que se configuran así. La primera parte reúne tres textos que, a manera de reflexiones epistemológicas, teóricas y metodológicas, aportan a la configuración del campo de conocimiento de la comunicación social. En la segunda parte, los artículos trascienden las reflexiones epistémicas para contar experiencias de investigaciones empíricas del uso de la técnica, en sus más variadas formas de aparición, tema obligado para los estudios de la comunicación, cuando ya desde Eco (1964), tenemos una lectura que resulta intensa al afirmar que el juego se establece entre apocalípticos e integrados. Aunque esta lectura hoy sugiere cierto reduccionismo, desde allí se inició el debate para pensar la comunicación masiva en relación con las hoy llamadas tecnologías de la información y la comunicación (TIC) y las discusiones que su uso suscita. La tercera parte contiene dos textos que priorizan la comunicación como proceso de interacción de la comunicación comunitaria, participativa e implicada. Investigación muy presente en el contexto latinoamericano.

\section{LAS CONTRIBUCIONES}

El primer conjunto de artículos trata sobre reflexiones epistémicas, teóricas y metodológicas, bajo la modalidad de ensayo o trabajo de corte teórico. Se trata de contribuciones que ponen el foco en la manera en que producimos conocimiento, en que ese proceso ocurre desde la alteridad, pues sin alteridad no hay cultura ni intercambio. No obstante, los señalamientos realizados por los autores también resaltan que ese carácter intersubjetivo en la construcción del conocimiento no está exento de las pujas de poder que se disputan fundamentalmente en los modos en que representamos a los otros.

Pedro Russi, docente e investigador de la Universidad de la República (Uruguay), inicia el dosier temático con "Epígrafes. Imaginaciones deseables y otras epistemes". El autor indica que "la gracia de un universo sin un centro claro es que depende de nosotros dónde ubicarlo y cambiar nuestra perspectiva". Su propuesta "no es dar conceptos o definiciones, sino compartir ideas como cuando estamos conversando", pues "imaginar futuros es ponernos a disposición para ser devorados por aquello que nos sorprende". "Sentipensar quiere decir e implica hacerlo desde el corazón, desde las entrañas, es visceral, con las tripas". Desde este posicionamiento, Russi nos plantea una serie de reflexiones que, si bien parten del ámbito educativo, cuestionan el sentido de la comunicación con minúscula o la Comunicación con mayúscula, en tanto "juego epistémico y lingüístico". A través de un texto cercano a prácticas alquímicas como la cultura, la interacción o la cocina: mezcla de invención, deseo y placer, nos propone la necesidad de comprender e imponernos la reinvención de la imaginación, el deseo, la palabra y la utopía de producir/pensar un nuevo espacio para la vida y sus vínculos activos de transformación. 
Continúan las reflexiones de este primer conjunto de contribuciones, las provenientes desde Brasil con el trabajo de Eloy Santos Vieira (Universidade do Vale do Rio dos Sinos) y Leila Lima de Sousa (Universidade Federal do Maranhão y Universidade do Vale do Rio dos Sinos), titulado "Epistemologias e descolonização na América Latina: compreendendo as mediações e a transmetodologia como práxis epistêmico-metodológicas transformadoras". Los autores ubican a la comunicación en diálogo con la cultura como el momento que constituye la institucionalización del campo de conocimiento. A partir de este hito, recuperan la figura metafórica de las "culturas bastardas" del investigador colombiano Omar Rincón, como punto de partida para "convocar mais algumas formas 'bastardas' de ver o mundo". Estas maneras de comprender lo que sucede en América Latina precisan de "processos de contraposição epistêmica, de desvinculação do genuíno ocidental, a partir da 'pluridiversidade' (Mignolo, 2008, p. 300)". Se trata de una toma de conciencia de nuestro mestizaje y del desarrollo de una "identidade política de resistência". Así los autores apuntan a la necesidad de reivindicar lo que llaman "epistemologias transformadoras" y los métodos que posibiliten el "diálogo com os sujeitos pesquisados, verificando que a experiência humana e os vários tipos de conhecimento devem estar unidos e serem a base da pesquisa em Comunicação social do século Xxı".

Por último, en este primer grupo de contribuciones se encuentra el texto de Eduardo César Augusto Muro Ampuero (Universidad de Bonn, Alemania), titulado "Vestigios coloniales en la representación del Otro: un reto para la comunicación decolonizadora". Los objetivos del trabajo recorren cómo a partir de una cierta forma de producción de conocimiento (con directas implicaciones en la historia como disciplina) se produce un paradigma en donde las representaciones de lo indígena ocupan un lugar subordinado en las relaciones intersubjetivas de las sociedades. También presenta algunos ejemplos sobre estos vestigios representacionales de lo indígena todavía presentes en la sociedad peruana, sobre todo, difundidos a través de los medios de comunicación; y, por último, busca reflexionar sobre lo anterior en el marco de una comunicación decolonizadora. El texto analiza el problema de las representaciones que se instalan como única verdad en los imaginarios ciudadanos y alude también al lugar de la comunicación de masas y el de la educación en las aulas. Lugares donde prevalecen los discursos y las representaciones del poder. La publicidad, como ejemplo del posicionamiento de estereotipos que hoy, más que nunca antes, vemos en las múltiples pantallas cómo reproducen estas narrativas patológicas y excluyentes. Se reclama una comunicación decolonizadora que cumpla con los principios básicos de respeto y el reconocimiento de la "otredad" como principio de generación de las identidades y de las diversidades. Se reflexiona acerca de que los medios de comunicación exploren a fondo las múltiples fuentes para ofrecer información densa en abordajes complejos y con sentido colectivo.

El segundo grupo de contribuciones se distingue de las anteriores porque se pasa de reflexiones epistémicas, con énfasis en las búsquedas teóricas y metodológicas, a experiencias de investigaciones empíricas y sistematizaciones de prácticas. El eje que 
vertebra a este segundo conjunto se asienta en las TIC y las discusiones en torno a las posibilidades que ofrecen, los obstáculos que se presentan en algunas poblaciones para su acceso y apropiación, así como los conflictos que se suscitan en el mundo del trabajo. Encontramos aquí contribuciones provenientes de Colombia, Brasil y Argentina.

Inaugura este grupo el trabajo de Tania Cobos de la Universidad Tecnológica de Bolívar, Colombia, el cual lleva por título "Las industrias periodísticas en la era de internet: el caso de los medios noticiosos colombianos en Google News Colombia". La llegada de la web, y la posterior aparición de las aplicaciones, también trajo consigo el surgimiento de nuevas formas de distribución del contenido noticioso de la mano de las compañías tecnológicas que incrementan exponencialmente su exposición a diferentes públicos y abaratan costos. Una de estas formas son los "agregadores de noticias". Un ejemplo de ellos es Google News, un servicio informativo propiedad de Google que cuenta con 72 ediciones para diferentes países y en diversos idiomas, así como con una dinámica de mejoramiento continuo, que también ha suscitado frecuentes y mediáticas polémicas con empresas periodísticas por su actividad. Este agregador rastrea la web; agrega los titulares (antes también incluía las primeras líneas), fotografías y videos de las noticias; y las categoriza y ordena dependiendo de su temática, fecha de publicación, medio noticioso, entre otros criterios. En este proceso no intervienen seres humanos, sino que es enteramente automatizado y ejecutado por un algoritmo. La práctica de agregación que hace Google News se realiza sin pedir permiso ni pagar licencias por los fragmentos que agrega, situación que ha sido fuertemente criticada por la industria de noticias en diferentes países. El artículo presenta los casos de dos medios colombianos que, frente al cuadro descrito, tuvieron reacciones diferentes, poniendo de manifiesto las controversias en el mundo del trabajo periodístico y los medios de comunicación.

De Colombia viajamos a Brasil, al "Encontro Online Cultura Livre do Sul: a produção cultural comunitária para a construção do comum" de Beatriz Cintra Martins (Universidade de São Paulo), Leonardo Feltrin Foletto (Universidade Federal do Rio Grande do Sul) y Carlos Eduardo Falcão Luna (Universidade Federal de Pernambuco). Este trabajo presenta la sistematización de un evento realizado íntegramente por medio de plataformas digitales, con el objeto de trascender la experiencia y poner de relieve discusiones en torno a cómo se construye lo común, tanto en el conocimiento como en las prácticas cotidianas. Los autores proponen que este diálogo entre saberes locales y culturas populares en red - que utiliza tecnologías de libre circulación- promueve a su vez la libre circulación de la cultura y del conocimiento. Mediante una metodología centrada en el análisis de contenido y la observación participante, esta investigación hace una apuesta por profundizar en lo que significan los encuentros de colectivos desde la perspectiva "libre" y la construcción de lo "común". Un reto que en el campo de conocimiento de la comunicación resulta sugerente para hablar del uso de tecnologías o del software libre, que en coherencia implica la reflexión acerca de este uso en contextos libres o grupos contraculturales y movimientos de resistencia que usan los soportes de libre acceso. 
En torno a las experiencias de investigaciones empíricas y con eje en las tecnologías, desde Brasil viajamos al noroeste argentino, a la provincia de Salta, con la presentación de María Rosa Chachagua, la cual se titula "Igualdad de posiciones para las juventudes rurales: educación y tecnología en la provincia de Salta (Argentina)". El análisis de la desigualdad estructural es enfocado a partir de un caso de educación mediada por tecnologías, y resulta una lectura de los procesos multidimensionales que se suscitan con la implementación de estas herramientas en entornos rurales. En la provincia de Salta, la desigualdad se materializa en la ausencia de servicios, de luz eléctrica, de agua potable, de vías de acceso a los territorios rurales, en el abandono sistemático. La autora aborda la problemática de jóvenes rurales vinculados a una educación que implementa la conectividad como solución para el acceso. Para ello, provoca encuentros en los que recoge testimonios tanto de profesores como de estudiantes y de trabajadores, para anclar las narrativas y la observación de las prácticas de educación y comunicación. Indaga por las proyecciones de vida en el futuro, un mundo mejor, el cual puede ser configurado desde unas prácticas sociales y de comunicación que así se lo propongan, en cuanto a gestionar la construcción colectiva.

Finalmente, el dosier se completa con dos trabajos que destacan el carácter vincular de la comunicación, el del cara a cara, ese que también es necesario para construir futuros deseables. Aquí las contribuciones tienen en común experiencias desarrolladas con comunidades donde prima la búsqueda de la participación y del involucramiento.

Por un lado, tenemos el aporte de Giovana Mesquita y Carolina Paz de la Universidade Federal de Pernambuco, Brasil, "Integrando comunicación y medicina en la enseñanza de la comunicación comunitaria: una experiencia brasileña innovadora". Las autoras abordan una experiencia de movilización social, ciudadana, participativa y contrahegemónica, la cual articula un exitoso proceso interdisciplinario en el nordeste brasilero, donde la comunicación comunitaria y la medicina se funden en una estrategia dialógica que expande el concepto de salud hacia otras apuestas más activas de transformación social, desarrollo humano y buen vivir en comunidad.

La ciudad de Caruaru, en el estado de Pernambuco, famosa por su tradición artesanal y festiva en toda la región, viene desarrollando un programa de innovación pedagógico y comunitario desde el año 2017 en el Centro Académico de Agreste, en alianza con el curso de comunicación de la Universidade Federal de Pernambuco. Un programa de enseñanza-aprendizaje, reflexivo y ético, que establece un vínculo directo entre la ciudadanía y las posibilidades que ofrece la comunicación en el desarrollo de nuevas dimensiones para producir y apropiar culturalmente un proyecto más amplio de la promoción de la salud, entendida esta como un bien social en clave de conciencia, derechos y participación ciudadana comprometida con la transformación colectiva de la realidad más vulnerable.

Por otro lado, está la contribución de Angie Tatiana Ramírez Rueda y Blanca Aracely López Rueda de la Universidad Pontificia Bolivariana de Colombia, la cual se titula 
"Audiovisual participativo: convergencia entre comunicación y medio ambiente". El trabajo sistematiza una experiencia de educomunicación con jóvenes, "Pescando historias. Memorias de un llanitero", quienes de forma colaborativa redescubrieron su territorio por medio de la fotografía, recuperaron las tradiciones orales de los pescadores con el uso de la radio y a través del audiovisual elaboraron diferentes narrativas acerca del cuidado de los recursos naturales. El trabajo se apoya en los lineamientos de la investigación acción (IA) dirigida a la transformación social en colaboración con la comunidad. Los audiovisuales resultantes fueron socializados tanto con los participantes de la estrategia como con los docentes de la institución educativa a la que asistían los jóvenes.

Como conclusiones, las autoras resaltan que la comunicación, si bien no logra transformaciones inmediatas en las comunidades, sí propicia espacios para que las personas asuman roles activos y juzguen desde su realidad eso que les afecta, pero al mismo tiempo propongan soluciones. La comunicación termina siendo ese espacio en el que convergen las ideas y desde el cual se construyen nuevos mensajes que alimentan el tejido social de las comunidades.

\section{COMUNICACIÓN PARA CONSTRUIR FUTUROS DESEABLES EN AMÉRICA LATINA}

Las contribuciones recibidas nos han dejado grandes enseñanzas en torno a esa construcción que llamamos "futuro". En primera instancia, que existen condicionantes tales como la desigualdad, la cual no se encuentra en abstracto, sino desde coordenadas definidas en términos de poblaciones, territorios, imposibilidades de acceso, apropiación y uso de tecnologías diversas (redes, medios de comunicación, procesos de participación). También señalan que en esa construcción presente y futura del campo de la comunicación sigue habiendo un compromiso irrenunciable en términos éticos y políticos, lo que se traduce en las maneras de construir el conocimiento, con quiénes lo hacemos, en las elecciones epistemológicas, teóricas y metodológicas que ensayamos en nuestros trabajos. Que el futuro tiene que ver con los deseos, con lo que las comunidades tienen para decir, con las movilizaciones, con la participación en distinta escala y que allí la comunicación, como actuación profesional y reflexión sobre la práctica, tiene para escuchar y aprender.

Antes de terminar esta presentación, queremos agradecer profunda y especialmente a los autores de las contribuciones, a los evaluadores y al equipo editorial de la revista Contratexto, por la dedicación, profesionalismo y voluntad de compartir que han mostrado en todo el proceso del dosier.

Finalmente, y retomando algunos de los trabajos, concebido el futuro como proceso de cambio, precisa del diálogo y no de la imposición. En términos de Follari (2019), a propósito de los sesenta años de CIESPAL y en un trabajo donde reivindica la política en el campo comunicacional, "no se trata de mirar al otro, sino de mirar con el otro más allá 
de ambos. Eso permite superar la particularidad de cada uno, la cual no se salva con la traducción y el entendimiento, sino solo con la constitución de un 'interés común' que esté por encima de los intereses particulares de cada sector interviniente (2019, p. 28)". Aunque estas sean horas de incertidumbres acerca del mundo tal como lo conocimos, el llamado es a no renunciar a la posibilidad de soñar, desear, imaginar el futuro para nuestras sociedades. No relegar nuestro protagonismo, sino reivindicarnos como activos y sentipensantes protagonistas del nuevo mundo que llegó.

Claudia Alejandra Kenbel Editora adjunta CONICET/Universidad Nacional de Río Cuarto, Argentina

Claudia Pilar García Corredor Editora adjunta Pontificia Universidad Javeriana, Colombia

\section{REFERENCIAS}

Eco, U. (1964). Apocalittici e integrati. Milán: Casa Ed. Valentino Bompiani.

Follari, R. (2019). ¡Que vuelva la política al análisis comunicológico! (consecuencias operativas de la epistemología aplicada). Revista Chasqui, 141, 17-29.

Kenbel, C., y García Corredor, C. P. (2019). Call For Papers, 33 (2020-1), Comunicación para imaginar futuros deseables en América Latina. Revista Contratexto. Recuperado de http://bit.ly/C4P_Ctx33-es

Mills, C. W. (1959). La imaginación sociológica. La Havana: Edición Revolucionaria. 